OPEN ACCESS

Edited by:

Antonio Pisani,

University of Pavia, Italy

Reviewed by:

Nurit Omer,

Tel Aviv Sourasky Medical

Center, Israe

Samir Abu-Rumeileh,

Ulm University Medical

Center, Germany

${ }^{*}$ Correspondence:

Guoyu Zhou

zhougy89@sdu.edu.cn

Specialty section: This article was submitted to Dementia and Neurodegenerative

Diseases,

a section of the journa

Frontiers in Neurology

Received: 26 April 2021

Accepted: 06 September 2021

Published: 06 October 2021

Citation:

Feng S, Zhao X, Zhou X, Ye X, Yu X,

Jiang $W$, Deng $Y$, Zhou $S, M a L$,

Shan $P$ and Zhou G (2021)

Epidemiological and Clinical

Characteristics of Sporadic

Creutzfeldt-Jakob Disease: A

Retrospective Study in Eastern China.

Front. Neurol. 12:700485.

doi: 10.3389/fneur.2021.700485

\section{Epidemiological and Clinical Characteristics of Sporadic Creutzfeldt-Jakob Disease: A Retrospective Study in Eastern China}

\author{
Shuo Feng ${ }^{1}$, Xinjing Zhao ${ }^{2}$, Xueying Zhou ${ }^{3}$, Xiang $\mathrm{Ye}^{2}$, Xiaolin $\mathrm{Yu}^{2}$, Wei Jiang ${ }^{2}$, Yu Deng ${ }^{4}$, \\ Shengnian Zhou ${ }^{1}$, Lin Ma ${ }^{2}$, Peiyan Shan ${ }^{2}$ and Guoyu Zhou ${ }^{2 *}$ \\ ${ }^{1}$ Department of Neurology, Qilu Hospital of Shandong University, Jinan, China, ${ }^{2}$ Department of Geriatric Neurology, Qilu \\ Hospital of Shandong University, Jinan, China, ${ }^{3}$ Department of Physical Medicine and Rehabilitation, Qilu Hospital of \\ Shandong University, Jinan, China, ${ }^{4}$ Department of Environmental Health, School of Public Health, China Medical University, \\ Shenyang, China
}

Objective: We aimed to characterize the epidemiological and clinical characteristics of sporadic Creutzfeldt-Jakob disease (SCJD) in eastern China in this retrospective study.

Methods: This study enrolled 67 patients with SCJD hospitalized in a grade-A tertiary hospital in eastern China from January 2010 to January 2020. Demographic data, clinical symptoms, brain magnetic resonance imaging (MRI), electroencephalogram (EEG), cerebrospinal fluid (CSF) 14-3-3 protein test, polymerase chain reaction (PCR), and DNA sequence determination of genes were collected and analyzed.

Results: There were 62 patients with probable sCJD and 5 patients with possible SCJD. Male (28 cases) to female (39 cases) ratio was 1:1.39. Mean age at disease onset was $64.42 \pm 9.00$ years (range: $29-88$ years), and mean survival time was 9.39 \pm 12.58 months (range: $1-60$ months for patients who received the follow-ups). The most common onset symptoms were dementia (49.25\%), movement disorder (44.78\%), and visual disturbance (22.39\%), while the most frequent clinical manifestations were language disorders (74.63\%), ataxia (70.15\%), and myoclonus (70.15\%). The positive rates of brain MRI abnormalities, 14-3-3 protein in CSF, and periodic sharp wave complexes (PSWCs) on EEG were 84.90, 68.00, and 46.03\%, respectively. The 14-3-3 protein positive $(p=0.033)$ and PSWCs on EEG $(p=0.020)$ acted as the favorable and unfavorable factor for over 1 year of survival time, respectively.

Conclusions: There were some differences in epidemiological and clinical characteristics among patients in China and those of other countries. The prognosis and its influencing factors were relatively unexplored in China. The mean survival time of Chinese patients was longer than that of Caucasian patients but shorter than that of Japanese patients. The 14-3-3 protein in CSF and PSWCs on EEG were both closely related to the survival time. It is necessary to promote autopsy or biopsy to better understand SCJD in China.

Keywords: prion disease, sporadic CJD, clinical, survival time, eastern China 


\section{INTRODUCTION}

Prion diseases (PrDs), also known as transmissible spongiform encephalopathies (TSEs), are a group of rare, rapidly progressive and fatal central nervous system diseases attacking humans and animals. According to the modes of human PrDs, PrDs can be classified into three different categories: sporadic (spontaneous), genetic (familial, inherited), and acquired (infectious, transmitted). The sporadic type, the most prevalent one (85-90\%), consists of sporadic Creutzfeldt-Jakob disease (sCJD), rare entities of sporadic fatal insomnia, and variably protease-sensitive prionopathy (VPSPr) $(1,2)$. sCJD is generally regarded as a spontaneous neurodegenerative illness, arising either from templated misfolding and protein conformation change of $\operatorname{PrP}^{\mathrm{C}}$ (normal brain prion-related protein) or spontaneous somatic mutation of PRNP (gene encoding the $\operatorname{PrP}^{\mathrm{C}}$ ). sCJD is clinically characterized by rapidly progressive dementia with ataxia, myoclonus, or other neurologic symptoms $(3,4)$ and neuropathologically represented by the presence of aggregates of abnormal prion protein, spongiform change, neuronal loss, and gliosis (5). At least six molecular pathological subtypes are classified by genotypes of PRNP codon 129 and protease cleavage sites of $\operatorname{PrP}^{\mathrm{Sc}}$ (misfolded disease-causing forms), which includes MM1, MV1, VV1, MM2, MV2, and VV2. They are responsible for the diversity of clinical characteristics like onset age, survival time, and symptoms $(6,7)$.

SCJD is a globally distributed disease. The identification of disease phenotype spectrum has greatly increased the possibility of early diagnosis of SCJD subtype specifically, which is also the foundation of developing effective treatments. In China, the CJD surveillance program supported by the Chinese Center for Disease Control and Prevention (CCDC) was conducted since 2006. sCJD cases (261) were reported nationwide from 2006 to 2010, with a higher number of cases in the east compared with those in other regions (8). However, the prevalence of SCJD has been rarely reported in the past decade in China. Besides, clinical features such as gender ratio and survival time are not consistent in several Chinese studies. Data on the prognosis of Chinese sCJD patients is even more deficient (8-14), and only one study had analyzed the factors affecting it (11). It is urgent to understand the current situation of SCJD in China. Here we presented a retrospective study on the epidemiologic and clinical characteristics and the prognoses of $67 \mathrm{sCJD}$ patients in eastern China to raise awareness of this rare neurodegenerative disease.

\section{MATERIALS AND METHODS}

\section{Patients Enrolled}

In this study, 67 suspected sCJD patients were enrolled from January 2010 to January 2020. All patients were admitted to the Department of Neurology, Qilu Hospital of Shandong University, which is a grade-A tertiary hospital in eastern China. Suspected SCJD cases were diagnosed according to the amended diagnostic criteria for CJD updated by Hermann et al. in 2018 (15). In brief, the possible diagnosis of SCJD is based on the clinical manifestations, which are progressive dementia and at least two out of four following signs: myoclonus, visual or cerebellar disturbance, pyramidal or extrapyramidal dysfunction, and akinetic mutism. The probable diagnosis of sCJD needs further clinical data, such as typical changes in EEG and MRI, or laboratory data, such as positive 14-3-3 protein in CSF. An absolutely definite diagnosis of any form of CJD usually requires either pathological or pathogenic examination of brain tissues. All patients had no history of blood transfusion, potential iatrogenic exposure from human cadaveric pituitary hormones, dura-mater implants, corneal grafts or neurosurgery. No patient had a family history of PrDs. Patients with diseases similar to sCJD, such as Hashimoto's encephalopathy, intoxication, Wernicke-Korsakoff syndrome, autoimmune encephalitis, and paraneoplastic syndrome, were excluded. This study was in compliance with the ethical principles of the Declaration of Helsinki and was approved by the ethical committee of the Qilu Hospital of Shandong University (KYLL-202011-189).

\section{Data Collection}

We examined the medical information and clinical records including demographic data, onset time, clinical manifestations, the results of neurologic physical examination, and specific auxiliary examinations. The following data were collected and analyzed: brain MRI scanning, electroencephalogram, cerebrospinal fluid 14-3-3 protein test, PCR and DNA sequence determination of PRNP gene from blood samples. Telephonebased follow-ups were carried out by professional neurological physicians to collect the information of disease outcome and survival time.

The neuropsychiatric symptoms of patients were defined as at least one of the following manifestations: agitation, depression, aggression, apathy, personality changes. Brain MRIs were performed using a 3.0-T superconducting magnet (GE Healthcare, Pittsburgh, PA, USA) and included T1, T2, fluid attenuated inversion recovery (FLAIR), and diffusion-weighted imaging (DWI) sequences. EEGs were performed by at least two technicians with relevant qualifications independently, and all cerebrospinal fluid samples were sent to the CCDC to detect 143-3 protein by Western blot. Survival time was calculated with the disease onset time and the death date.

\section{Statistical Analysis}

All data were analyzed using SPSS 26.0 software (IBM Corp., Armonk, NY, USA). Descriptive statistics were performed on the demographic characteristics. Continuous variables were presented in the form of mean \pm standard deviation (SD) and compared by Student's $t$-test. Categorical variables were expressed as frequency or percentage, while comparison was done with Chi-square test and Fisher's exact test. KaplanMeier survival analysis was conducted for deceased patients. Cox proportional hazards regression model and forest plot were applied to describe and analyze the survival time. Statistical significance was set at $p<0.05$.

\section{RESULTS}

In the past 10 years, the number of clinically confirmed sCJD cases in our center is on the rise generally, reaching the highest 


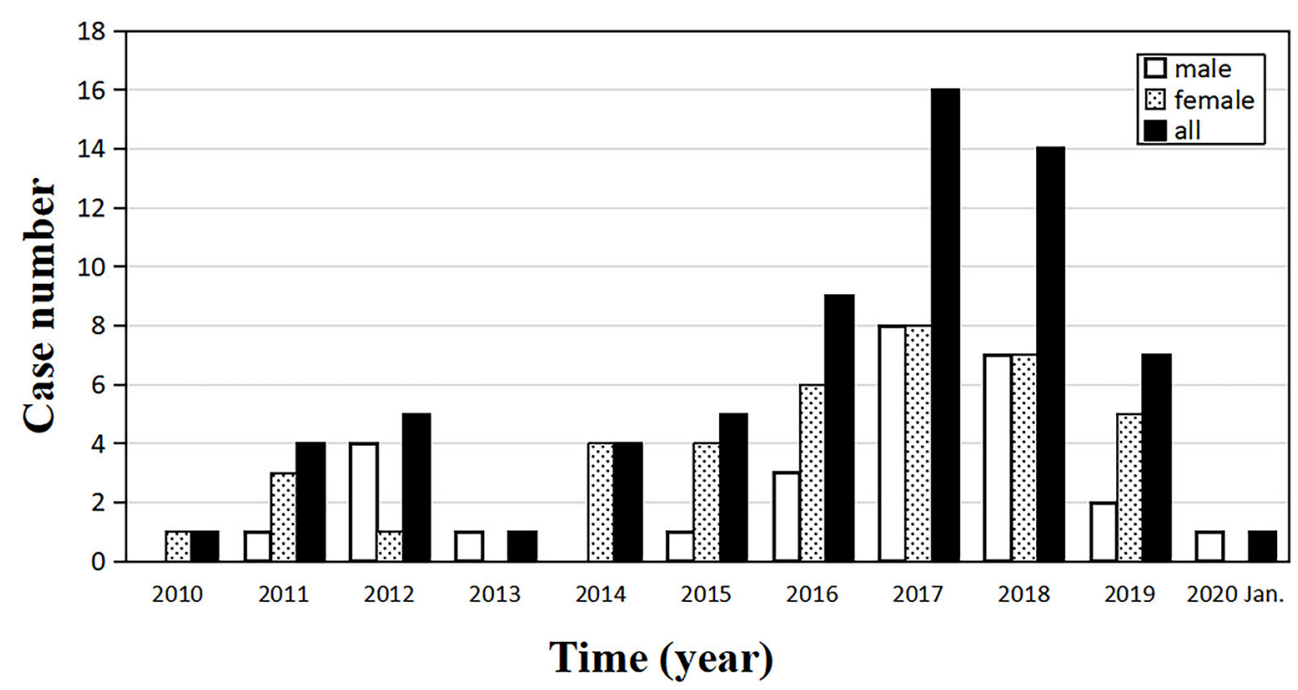

FIGURE 1 | Annual distribution of the number of sporadic Creutzfeldt-Jakob disease (sCJD) patients, from January 2010 to January 2020 ( $n=67)$.

level of 16 in 2017 (Figure 1). At the end of the study, 43 cases were followed up and 39 cases died.

\section{General Demographic Features}

Out of 67 enrolled cases, 62 were probable diagnosis of sCJD, and 5 cases were possible diagnosis of sCJD. There were 39 females and 28 males, with a gender ratio of 1.39:1. The age of onset ranged from 29 to 88 years (mean \pm SD: $64.42 \pm 9.00$ years, median: 66 years). The peak of age groups was $65-69$ years $(31.34 \%)$ in all patients, and the same pattern could be observed in the female subgroup. For males, the peak was 60-64 years, earlier than females (Figure 2). The mean $( \pm S D)$ and median onset ages of males was $63.57 \pm 6.90$ and 64 years, respectively (range: 46-75 years). No significant difference was found in the mean age $(p=0.519)$ and the median age $(p=0.212)$ between the male and female groups (Table 1).

\section{Clinical Features}

Dementia (49.25\%), movement disorder (44.78\%), and visual disturbance $(22.39 \%)$ were the most common onset symptoms in 67 patients. Visual disturbance was more predominant in females than in males ( $p=0.043$ ) (Table 2). Neuropsychiatric symptoms, dizziness, epilepsy seizure, sleep disorders, and language disorders were also observed at the onset of disease. The most common clinical manifestations were language disorders (74.63\%), ataxia $(70.15 \%)$, and myoclonus (70.15\%). Patients with movement disorder in this study all manifested as parkinsonism characterized by bradykinesia (53.73\%), and two of them had static tremor simultaneously (2.99\%). Moreover, $58.21 \%$ of the patients developed neuropsychiatric symptoms, with psychotic symptom (26.87\%) as the most common one, followed by apathy (19.40\%) and panic (13.42\%). Akinetic mutism occurred in $37.31 \%$ of the patients (Table 2).

\section{Brain Magnetic Resonance Imaging}

Sixty-six patients underwent brain MRI scanning, and 56 (84.85\%) of them had typical MRI abnormalities, such as "cortical ribboning," abnormal signal in basal ganglia on DWI. Among them, 50 cases $(75.76 \%)$ had "cortical ribboning," 29 cases (43.94\%) showed abnormal signal in basal ganglia (Table 2), and 23 cases $(34.85 \%)$ had both. Twenty-seven cases $(40.91 \%)$ had only "cortical ribboning," while six cases (9.09\%) presented abnormalities only in basal ganglia.

\section{Electroencephalogram}

Of the 63 patients who undertook EEG examinations, 29 had PSWCs (positive rate: $46.03 \%$ ), including 7 males and 22 females. Statistical analyses showed a significant difference in the positive rates of PSWCs between male and female groups, and the female group had a higher positive rate $(P=0.006)$ (Table 2$)$.

\section{4-3-3 Protein}

A total of 25 patients, including 12 males and 13 females, were tested for cerebrospinal fluid 14-3-3 protein. Seventeen patients were positive (positive rate: $68.00 \%$ ). The positive rate for males and females was $75.00 \%$ (nine positive cases) and $61.54 \%$ (eight positive cases), respectively (Table 3). Meanwhile, we managed to complete the follow-ups for 17 of the tested patients, among whom 15 cases were 14-3-3 protein positive. Four patients, whose survival time was more than 24 months, were still alive. We explored the relationship between 14-3-3 protein and other three factors including gender, age, and survival time. Survival time for patients with positive CSF 14-3-3 protein (8.55 \pm 6.06 months, median 7 months) was significantly longer than that for patients without $14-3-3$ protein ( $2.00 \pm 0$ months, median 2 months) ( $p$ $=0.005)$ (Table 3 ). 


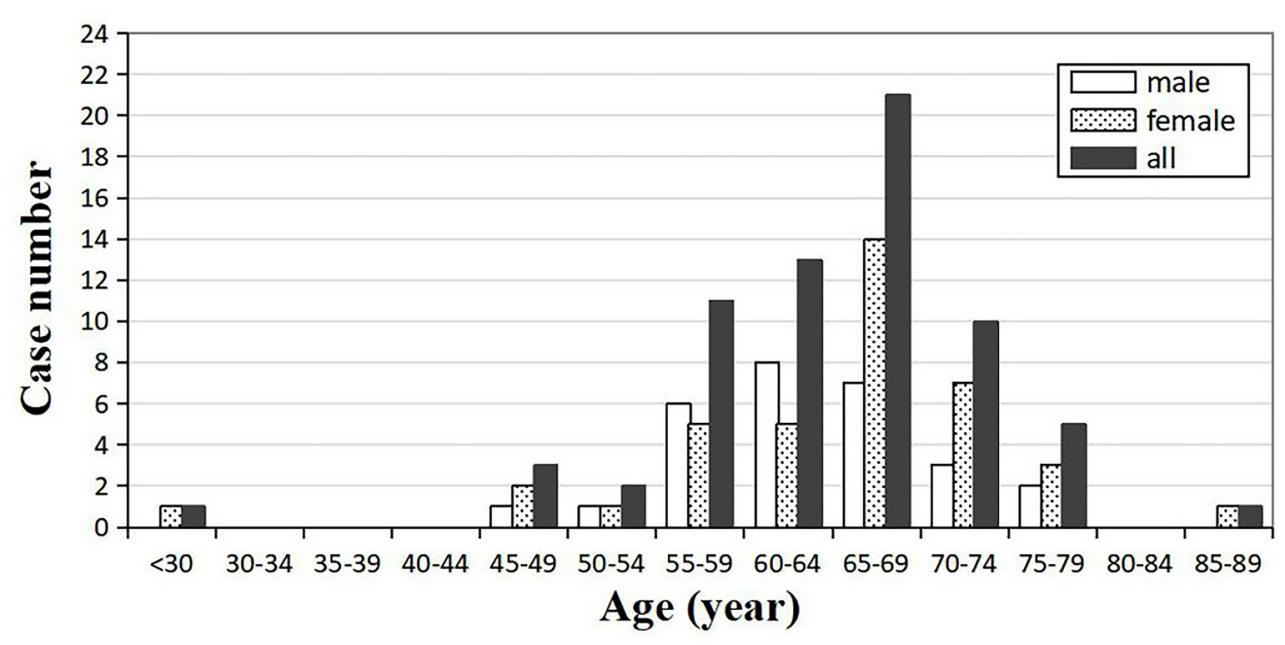

FIGURE 2 | Distribution of the onset age and gender-specific onset age in SCJD patients $(n=67)$.

TABLE 1 | Onset age and survival time of sporadic Creutzfeldt-Jakob disease (sCJD).

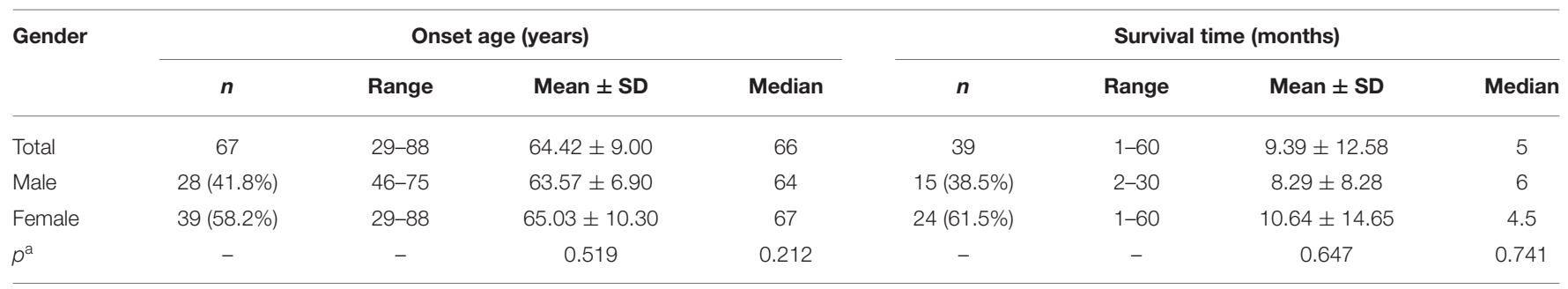

${ }^{a}$ Difference in mean onset age and survival time between two genders were analyzed by Student's t-test method. Difference in median onset age and survival time between two genders were analyzed by Brown-Moo test method.

\section{PRNP Gene}

PCR and DNA sequence determination of PRNP gene were performed in $25 \mathrm{sCJD}$ patients ( 12 males and 13 females) in our study. No PRNP gene mutation was found in these 25 cases and 24 of them had the methionine homozygous genotype at codon 129 (MM, 96.00\%). Only one was heterozygous for methionine/valine (MV, 4.00\%).

\section{Survival Time}

Forty-three patients (19 males and 24 females) received the follow-ups, and 39 patients died. The onset age of the 43 patients was from 47 to 78 years. In each subgroup based on age, most patients lived $<12$ months (Figure 3 ). Thirty-one cases (12 males and 19 females) died within 12 months (72.09\%), five cases (2 males and 3 females) had a survival time between 13 and 24 months (11.63\%), and seven cases (4 males and 3 females) were still alive 24 months after the onset (16.28\%) (Figure 4). The mean survival time was $9.39 \pm 12.58$ months, and the median was 5 months (range: 1-60 months) (Table 1).

Kaplan-Meier survival curves for the patients who underwent the follow-ups are shown in Figure 5A (subgroups by gender) and Figure 5B (subgroups by age). No statistical difference of survival time was observed between the groups of different genders $(p=0.228)$ (Figure 5A). The median survival time of patients under 60 years old was 7 months, while that of patients over 60 years old was 8 months. The difference in survival time was not statistically significant between different age groups ( $p=$ 0.667), either (Figure 5B). Cox proportional hazards regression model was used to analyze factors influencing the survival time. The results showed that 14-3-3 protein in CSF was favorable to over 1 year of survival time, while the PSWCs on EEG was an unfavorable factor for surviving over 1 year after onset (Figure 6).

\section{DISCUSSION}

In the current study, we retrospectively analyzed the demographic and clinical features of 67 sCJD patients in eastern China, and 43 of them received follow-ups. By the end of the study, 39 follow-up patients had died. Generally speaking, though PrDs are considered rare, the number of sCJD cases diagnosed in our hospital has been on the rise over the past 10 years. This may be attributed to the aging population, increasing number of patients admitted to hospital, and improved clinician awareness of the disease. However, the number of cases peaked in 2017 and has since declined mildly. It is for sure that the total number of inpatients from the department of neurology in our hospital has been increasing from 2010 to 2019. Given that the number of CJD cases nationwide in the recent 5 years has 
TABLE 2 | The main clinical and ancillary test features of SCJD patients.

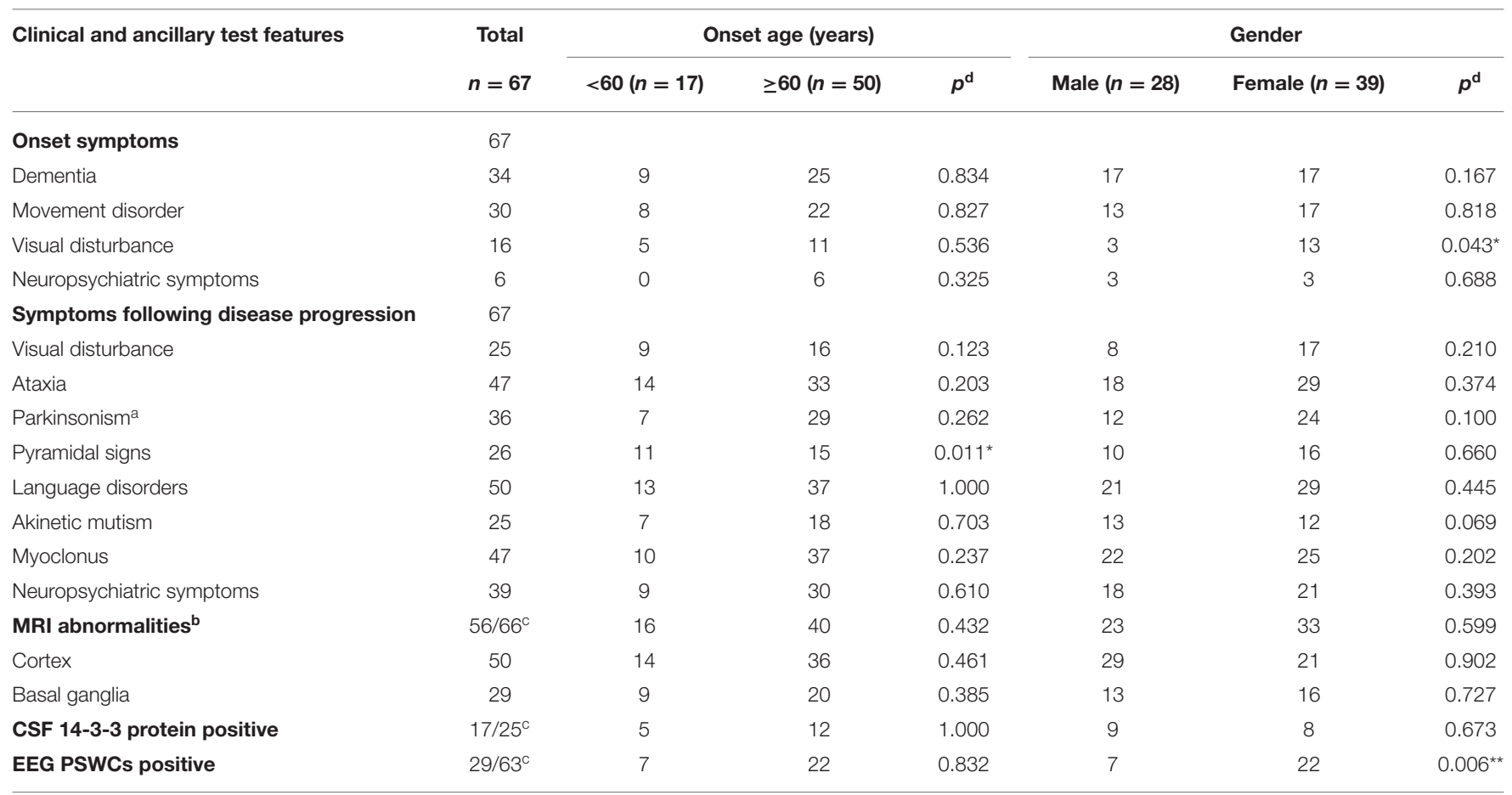

${ }^{a}$ Bradykinesia, static tremor, or both.

${ }^{b}$ Diffusion-weighted imaging brighter than fluid-attenuated inversion recovery.

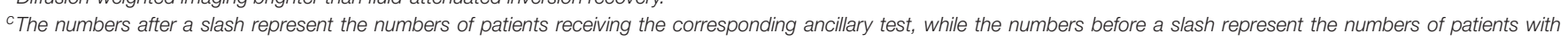
positive results.

${ }^{d}$ Chi square test was used to compare the features of the two groups.

" $p<0.05 ;{ }^{* *} p<0.01$.

CSF, cerebrospinal fluid; EEG, electroencephalogram; PSWCs, periodic sharp wave complexes.

TABLE 3 | The 14-3-3 protein in cerebrospinal fluid (CSF) of SCJD patients.

\begin{tabular}{|c|c|c|c|c|c|c|c|c|c|}
\hline \multirow[t]{2}{*}{$14-3-3$} & \multicolumn{4}{|c|}{ Gender $(n=25)$} & \multicolumn{2}{|c|}{ Onset age (years, $n=25$ ) } & \multicolumn{3}{|c|}{ Survival time (months, $n=13$ ) } \\
\hline & $n$ & Male & Female & $p^{b}$ & Mean \pm SD & Median & $n$ & Mean \pm SD & Median \\
\hline Positive & 17 & 9 & 8 & 0.896 & $65.29 \pm 7.05$ & 67 & 11 & $8.55 \pm 6.06$ & 7 \\
\hline Negative & 8 & 3 & 5 & - & $65.63 \pm 10.32$ & 67.5 & 2 & $2.00 \pm 0$ & 2 \\
\hline$p^{\mathrm{a}}$ & - & - & - & - & 0.926 & - & - & $0.005^{\star}$ & - \\
\hline
\end{tabular}

${ }^{a}$ Differences in mean age and mean survival time between two groups (14-3-3 positive and 14-3-3 negative) were analyzed by Mann-Whitney U-test method.

${ }^{b}$ The relationship between 14-3-3 protein positive and gender was analyzed by Spearman correlation analysis method.

* $p<0.01$.

not been published, and other Chinese single-center study have not paid attention to the trend of the number of the patients, whether this trend is consistent with the national situation remains unknown.

In this study, we found that there were more female than male sCJD patients, and the female-to-male ratio was 1.39. Two studies of Chinese patients were consistent with our findings, in which the number of females predominated $(10,12)$, but two other studies had reported a higher proportion of males, where the female-to-male ratio was 0.82 and 0.79 , respectively $(8,14)$. Because of small sample size and various nationalities in China, there might be biases in those results. A convincing gender ratio of sCJD patients in China requires demographic data of multicenter or population-based studies in the future. According to Japanese national SCJD surveillance data from 2001 to 2010, the ratio of female to male was 1.15 (16). In a research with 2,451 pathologically confirmed SCJD patients enrolled by the European Creutzfeldt-Jakob Disease Network (EUROCJD), which covered several European countries, Canada, and Australia, the ratio of female to male was 1.18 (7). In contrast, the data of $116 \mathrm{sCJD}$ cases from the United States demonstrated that the female-to-male ratio was 0.49 (17). It is worth noting that this American study included patients of multiple races including Whites, Blacks, and American Indians. 


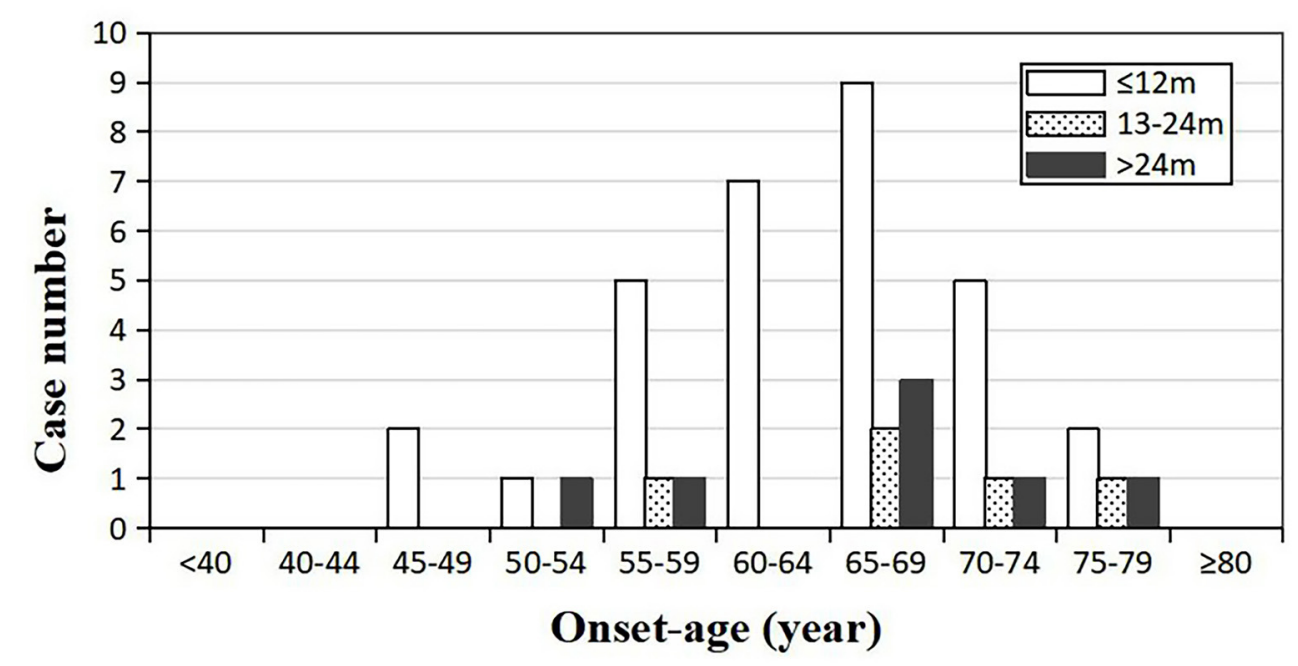

FIGURE 3 | Distribution of the survival time among SCJD patients stratified by onset age $(n=43)$. Across all age groups, patients whose survival times were $<1$ year tend to be the most, indicating that the survival time may not be necessarily related to the onset age.

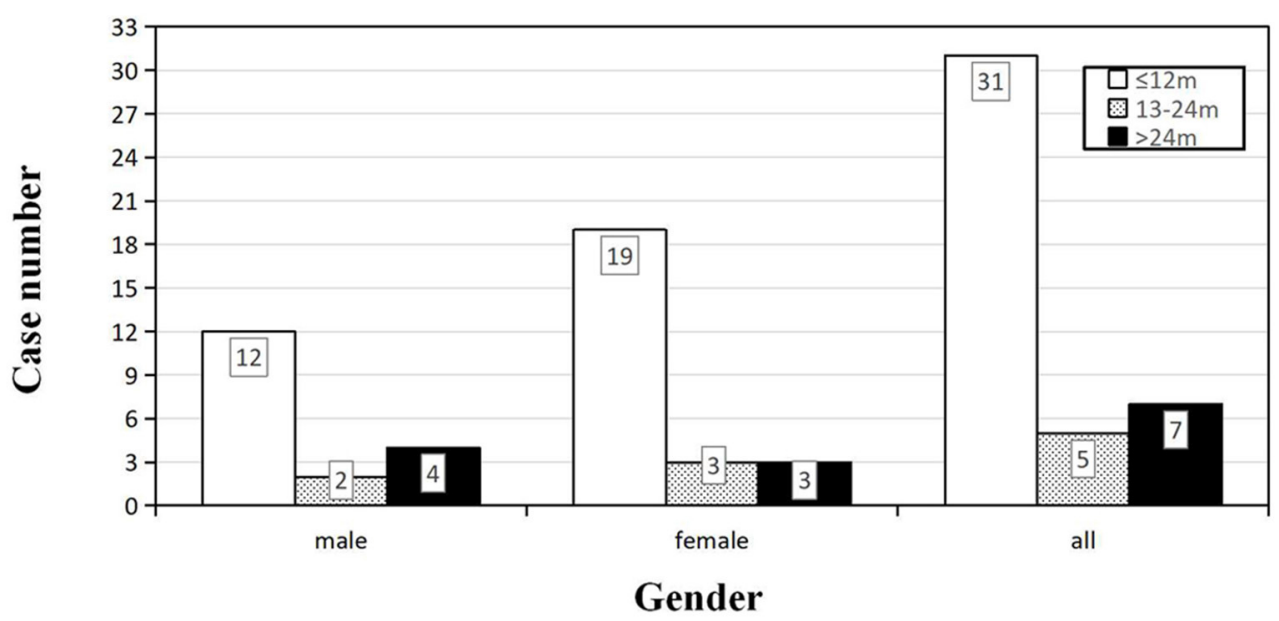

FIGURE 4 | Distribution of the survival time among SCJD patients stratified by gender $(n=43)$. For both male and female, the largest proportion of patients had a survival time within 1 year, indicating that the survival time may not be necessarily related to the gender.

Race, population structure, molecular pathological subtype pattern, and the gender difference in economic status could partly contribute to the disparate ratios by countries. Further researches are required to investigate the exact reasons by controlling multiple factors. What is more, adequate sample size is indispensable in that the patient numbers of Chinese studies are much less than that in other countries.

The mean age of disease onset in this study (64.42 years old) was comparable with the findings from Korea (65.5 years) (18), Japan (65.5 years) (19), the United States (66.15 years), (20) and Europe (66 years) (21), but higher than that of a few Chinese patient groups [58 years (14) 60.3 years (9)]. Another two surveys in China have produced results essentially in agreement with ours at the median onset age, which was
64 years in our study $(8,11)$. According to these reported Chinese studies, most of suspected patients were not tested for PRNP gene to rule out genetic Creutzfeldt-Jakob disease $(8,9,11,14)$, which might affect the results and analysis of the study. Similarly, there were two patients younger than 30 years old in our study. Although these two patients met the criteria for clinical diagnosis of sCJD, they were not eventually diagnosed by pathological examination, which may also have an impact on the result. At the same time, considering that the different regional demographic features might play a role in the heterogeneity of onset ages among domestic studies, more accurate onset age remains to be verified by large national surveys. Additionally, Kotkowski et al. found that age (66 \pm 11.5 years) was correlated with sCJD onset (20). As with 

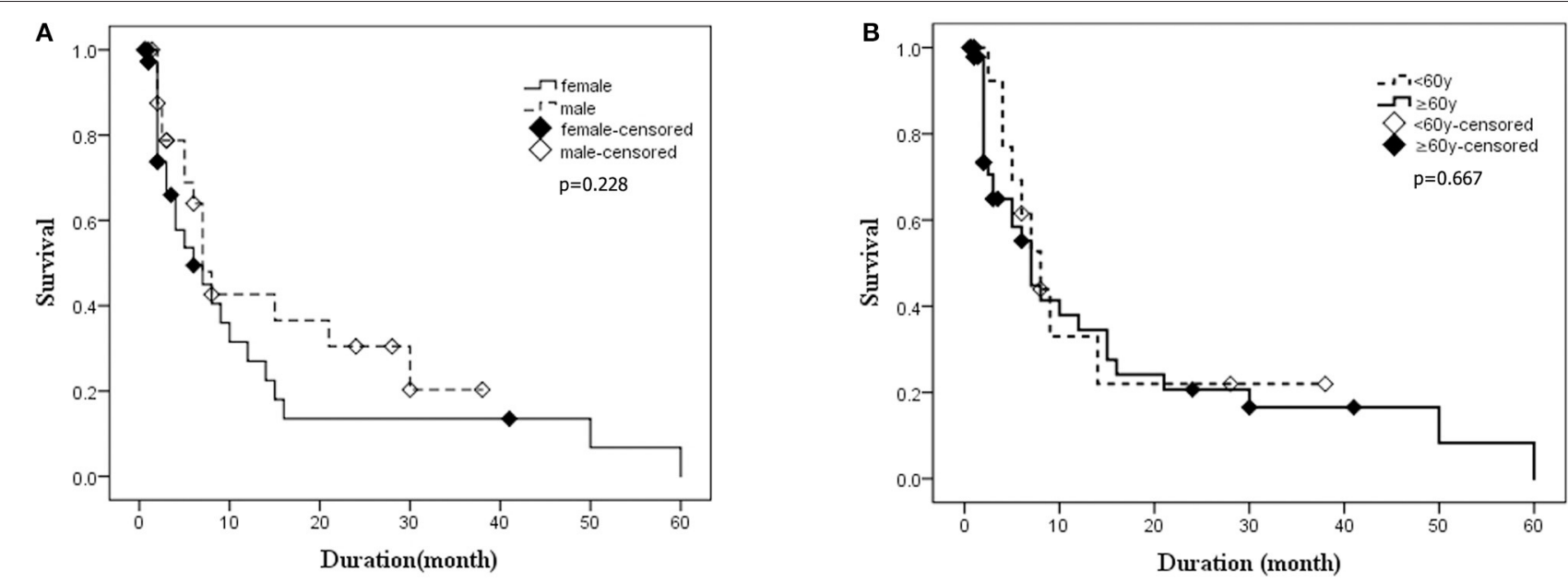

FIGURE 5 | The Kaplan-Meier survival curves for deceased patients. The X-axis represents survival time (months), and the Y-axis represents survival probability. (A) Survival time for patients stratified by gender. (B) Survival time for patients stratified by age. Graphic symbol shows the median survival time (50\% percentile) of the distinct gender or age groups.

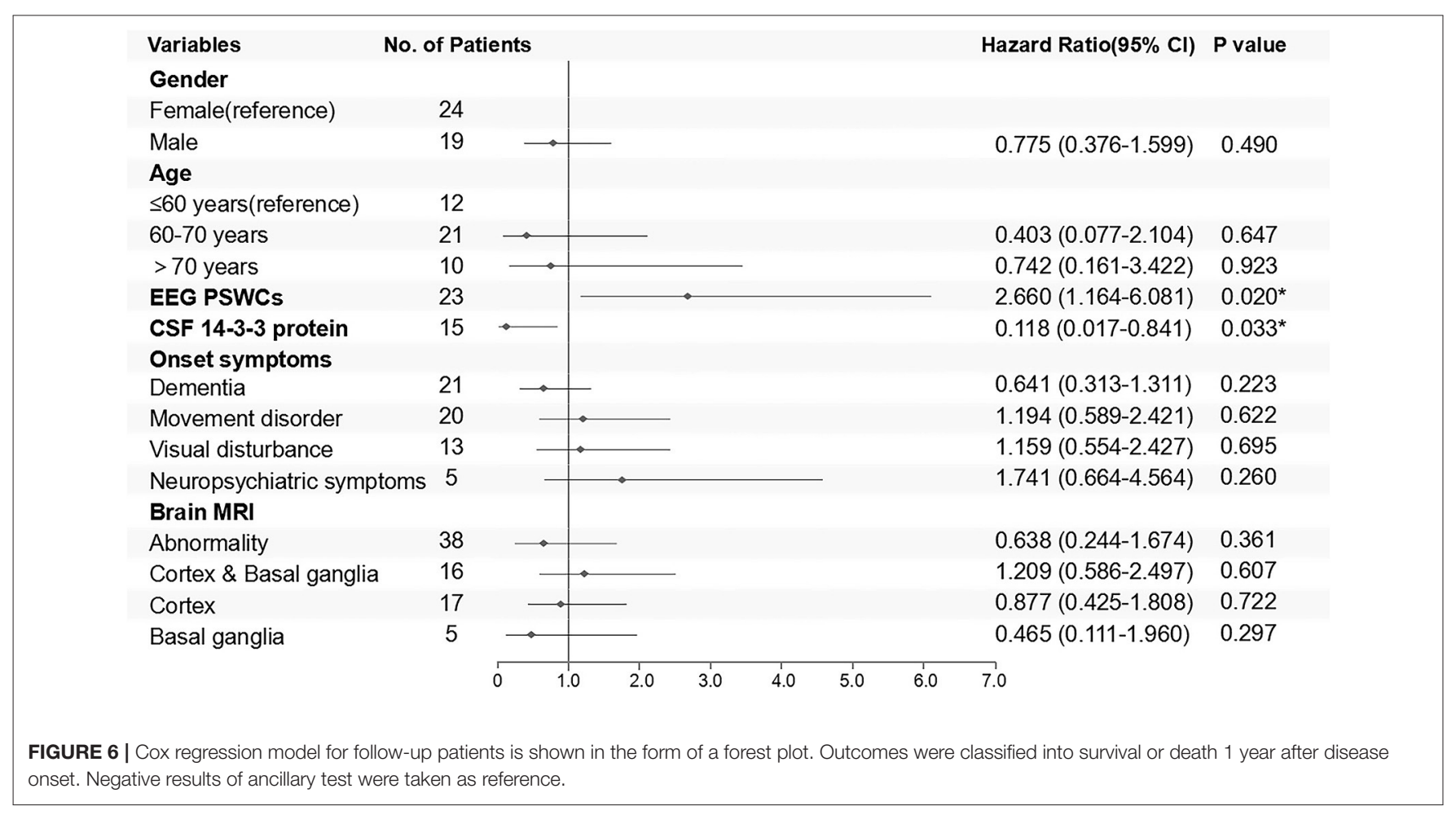

many other neurodegenerative diseases, sCJD generally occurs in late adulthood.

So far, no effective treatment has been developed for sCJD, which remains uniformly fatal. In our study, the mean survival time was 9.39 months, and $72.09 \%$ of the sCJD patients had a survival time $<1$ year. Similarly, other researches in China showed that mean survival time ranged from 6.1 to 11.6 months, and 1-year mortality was $68.50-74.00 \%(11,13,14)$. We also found that 14-3-3 protein and PSWCs acted as the favorable and unfavorable factor for over 1 year of survival time, respectively. The same predictive effect of PSWCs was also observed in two European large-scale studies, in which over 2,000 pathologically definite SCJD cases were incorporated $(7,22)$. However, some studies have found that 14-3-3 protein was correlated with a shorter survival time $(7,21-24)$. The possible reasons for this discrepancy may be as follows: first of all, there are only two 14-3-3 protein-positive patients in this study, whose survival times were $<1$ year. Considering the 
small sample size and related uncertainties, our findings need to be further validated by large-scale studies. Second, the survival time is usually not determined by a single factor, which is why Llorens et al. have set up a multifactor predictive model (21). To our knowledge, previous study objects were Caucasians, but no Chinese researchers have explored the relationship between CSF biomarkers and survival time. Different races, regional environments, molecular subtypes and some relevant unknown factors may partly explain our different findings. Last but not the least, pathological examinations were not carried out in our patients, which could reduce the accuracy of diagnoses and then affect the results. Pocchiari et al. concluded that there was correlation between survival time and codon 129 type (22). The multinational study carried out by EUROCJD, whose research objects were mainly Caucasians, revealed that the mean survival time was only 5 months, and $85.8 \%$ of the cases died within 1 year. The MM, MV, and VV type of codon 129 accounted for 66.1, 17.0, and $16.9 \%$, respectively (7). In Asia, almost all the sCJD patients were MM type $(8,9,11,14,25-27)$. MM type accounted for 97.0-100.0\% of all sCJD patients according to previous Chinese studies, while others were all MV type $(8,9,11,14,27)$, which was in agreement with our findings. However, due to the extreme paucity of pathological examination, $\mathrm{PrP}^{\mathrm{Sc}}$ types of Chinese $\mathrm{SCJD}$ patients are unavailable till now. As a consequence, different distribution patterns of PRNP codon 129 types may be one of the reasons for the variations in survival time, and other possible reasons remain to be explored.

However, according to a Japanese report, the mean survival time and 1-year mortality was 15.7 months and 48.1\%, respectively (26). By comparison, survival time of the patients is shorter in China, where the MM type is the majority in Japan, though $(8,11,14,26)$. It should be noted that there is a proportion of Japanese patients with MM2 type, and nearly all of those survival time was more than 1 year, and some even reached 6 years (28). Additionally, the MM2 type can be further divided into MM2C and MM2T subtypes based on distinctive cortical and thalamic histopathology $(2,29)$, and the MM2T subtype is also known as sporadic familial insomnia ( $\mathrm{sFI}$ ), which is relatively rare. Their mean survival time was comparable despite different races $(28,30)$. Studies also have shown that MM1 type patients had an obviously shorter media survival time of 4.0-9.0 months $(7,31)$. However, the pathological classification of MM type is still not clear in China, which might be the main reason for the different survival time between Japanese and Chinese patients. More pathological results are needed to verify it. What is more, Iwasaki et al. found that tube feeding was an independent factor influencing total disease duration for MM1 type of sCJD (31), which also partly accounted for the longer survival time of Japanese patients (32). Future studies should be conducted by controlling possible interfering factors, such as regions, economic conditions, races, or molecular pathological subtypes, to find the exact factors that affect the course of disease.

The most common initial symptoms in our study were dementia, movement disorder, and visual disturbance, which were consistent with the results of other two centers in China $(9,11)$. It was reported by a Japanese research that $55.10 \%$ of cases started from psychiatric symptoms such as dementia or sleeping difficulties (26). According to a German study of 492 sCJD patients, dementia was the most common initial symptom followed by cerebellar, visual, and psychiatric disturbances (33). An American study with 114 sCJD patients showed that cognitive symptoms, especially memory loss, were the most common manifestations (34). Despite the discrepancies in race, region and even molecular pathological types, dementia is the most frequent onset symptom in these patients. Most notably, $49.25 \%$ of our patients rapidly developed progressive dementia, almost all of whom had this symptom at the time of seeking medical treatments.

The diagnosis of sCJD is challenging. Only autopsy can make a definite diagnosis, but most diagnoses of sCJD are based on clinical manifestations and ancillary tests without specific biological markers. Biomarkers with high specificity and sensitivity are urgently needed to assist clinicians in diagnosing sCJD. The first biomarker identified for diagnosis was PSWCs on EEG, which was performed in 63 patients in our study. Only $29(46.03 \%)$ of them showed positive results, but the sensitivity of PSWCs among the confirmed CJD patients was $55.7-64 \%(7,35)$. Since PSWCs usually occur in the late stages of the disease, the timing of test can also affect the positive rates. Serial EEG recordings are required for higher positive rates (36-38). Furthermore, given that PSWCs are common in encephalopathies, metabolic disorders, dementias, and other diseases, the specificity is relatively low $(35,39-41)$. Another widely used biomarker was 14-3-3 protein in CSF. As a moderately sensitive biomarker (42), 14-3-3 protein showed a positive rate of $68.00 \%$ in our cases, not very prominent detecting efficiency. Compared with PSWCs and 14-3-3 protein, brain diffusion-weighted MRI has a higher diagnostic utility in sCJD, with a sensitivity ranging from 92 to $96 \%$ and a specificity of about 93 to $94 \%(43-45)$. Further studies indicated that specific MRI abnormalities could serve as useful parameters for predicting the clinical course, such as those who have cortical plus basal ganglia hyperintensity on DWI tend to show symptoms of advanced stage $(46,47)$. The abnormal rate of brain MRI of our patients was $84.85 \%$, which was a bit lower than that of studies with some defined patients $(10,42-44)$. One of the possible reasons is that our clinical diagnosis of SCJD is not conclusive without pathological inspections. In recent years, some of the newer diagnostic markers have also been explored; total $(\mathrm{t})$-tau protein in CSF was found to be superior to 14-3-3 protein and neurofilament light chain protein (NfL) as a biomarker with the highest sensitivity of $88.2-90.20 \%$ (48) and specificity of 74.5$78.9 \%(49,50)$. The ratio of t-tau and phosphorylated-tau may be useful to distinguish patients with CJD from patients with other dementias $(51,52)$. In addition, a new $\mathrm{PrP}^{\mathrm{Sc}}$ amplification assay, named real-time quaking-induced conversion (RT-QuIC), has also shown an overall diagnostic sensitivity of $82.1-92 \%$ and a specificity of $98.5-100 \%(49,53-56)$, sometimes behind t-tau. Unfortunately, none of our patients had received the above two tests on account of technical limitations and examination costs. These problems could change with the deepening understanding and research of the disease.

Compared with nearly $100 \%$ in Austria (57), 68.7\% in the United States (58), 66\% in Germany (59), 60\% in Australia 
(60), and $51 \%$ in Belgium (61), the autopsy rate is extremely low among Chinese patients due to the influence of traditional Chinese values. Besides, the fear and limited understanding of prion disease, as well as the restriction on the conditions of medical institutions, also affect autopsies on patients with this contagious neurodegenerative disease in China. Biopsy could not be performed smoothly and generally in China, either, which was confirmed by previous domestic literature $(8,10-13)$. Thus, their vast majority of molecular pathological subtypes remained unknown. To have a more accurate understanding of SCJD in China, the application of autopsy or biopsy in suspected patients is urgently needed.

This study described and analyzed the epidemiological and clinical characteristics of sCJD patients in eastern China systematically. Compared with most previous studies in China, we have gotten a relatively detailed clinical characteristics and complete follow-up data, and conducted a thorough analysis of the relationship between the two. Moreover, in the absence of autopsy and biopsy, compared with to the only study that performed prognostic analysis (11), the newer clinical diagnostic criteria were applied here. Differences in gender, 14-3-3 protein, PSWCs, and contributing factors on survival time were revealed in Chinese patients for the first time. There are also several limitations in our study. First, the number of sCJD cases was relatively small, and CSF 14-3-3 protein and PRNP gene analysis were not performed in all cases. With the ongoing deep research work, the rate of CSF and gene testing for patients with prion disease would increase gradually, thus, raising the accuracy of sCJD diagnosis. Second, some patients were lost to followup, resulting in unknown prognoses. In future research, we will expand the sample size and conduct more intensive and longer-term follow-up observations. Third, although the primary diagnostic methods for sCJD are t-tau and RT-QuIC, rather than the 14-3-3 protein and PSWCs now, the first two were not performed in this study. Finally, pathological examination of brain tissue, which is crucial to diagnosis, was not carried out. Along with the further understanding of prion disease and the development of medical and economic conditions in China, this situation is expected to be improved.

\section{CONCLUSIONS}

In conclusion, the epidemiological and clinical characteristics in Chinese studies are inconsistent, and there are some differences when compared with other countries. The mean survival time of

\section{REFERENCES}

1. Masters CL, Harris JO, Gajdusek DC, Gibbs CJ, Jr., Bernoulli C, et al. Creutzfeldt-Jakob disease: patterns of worldwide occurrence and the significance of familial and sporadic clustering. Ann Neurol. (1979) 5:177-88. doi: 10.1002/ana.410050212

2. Puoti G, Bizzi A, Forloni G, Safar J, Tagliavini F, Gambetti P. Sporadic human prion diseases: molecular insights and diagnosis. Lancet Neurol. (2012) 11:618-28. doi: 10.1016/S1474-4422(12)70063-7
Chinese patients was longer than that of Caucasian patients but shorter than that of Japanese patients. This study found that 143-3 protein in CSF and PSWCs on EEG acted as the favorable and unfavorable factor for over 1 year of survival time of Chinese patients, respectively, which has not been reported in previous literature of China. With an extremely low autopsy rate of prion disease in China, it is necessary to promote autopsy or biopsy to better understand the incidence, clinical characteristics, and the effective factors for prognosis of sCJD or prion diseases. Systematic studies on genes, protein, pathology, and clinical phenotypes are needed for a deeper insight of this disease as well as other possible neurodegenerative diseases related to protein misfolding.

\section{DATA AVAILABILITY STATEMENT}

The raw data supporting the conclusions of this article will be made available by the authors, without undue reservation.

\section{ETHICS STATEMENT}

The studies involving human participants were reviewed and approved by the Research Ethics Commission of Qilu Hospital of Shandong University, Jinan, China (KYLL202011-189). Informed consent was waived by the Research Ethics Commission.

\section{AUTHOR CONTRIBUTIONS}

GZ designed the study and reviewed the manuscript. GZ, XZha, XYe, XYu, XZho, WJ, SF, SZ, PS, and LM collected the data. GZ, SF, and YD performed the statistical analysis. SF summarized the data and drafted the manuscript. All authors were responsible for the final approval of the version to be published.

\section{FUNDING}

This study was supported by the Key Research and Development project of Shandong Province, China (Grant No. 2019GSF108230).

\section{ACKNOWLEDGMENTS}

The authors are very grateful to the patients who participated in this project.

3. Will R, Matthews W. A retrospective study of Creutzfeldt-Jakob disease in England and Wales 1970-79. I: Clinical features. J Neurol Neurosurg Psychiatry. (1984) 47:134-40. doi: 10.1136/jnnp.47.2.134

4. Brown P, Cathala F, Castaigne P, Gajdusek DC. Creutzfeldt-Jakob disease: clinical analysis of a consecutive series of 230 neuropathologically verified cases. Ann Neurol. (1986) 20:597-602. doi: 10.1002/ana.410200507

5. Parchi P, Strammiello R, Giese A, Kretzschmar H. Phenotypic variability of sporadic human prion disease and its molecular basis: past, present, and future. Acta Neuropathol. (2011) 121:91-112. doi: 10.1007/s00401-010-0779-6 
6. Parchi P, Giese A, Capellari S, Brown P, Schulz-Schaeffer W, Windl O, et al. Classification of sporadic Creutzfeldt-Jakob disease based on molecular and phenotypic analysis of 300 subjects. Ann Neurol. (1999) 46:224-33. doi: 10.1002/1531-8249(199908)46:2<224::AID-ANA12>3.0.CO;2-W

7. Collins SJ, Sanchez-Juan P, Masters CL, Klug GM, van Duijn C, Poleggi A, et al. Determinants of diagnostic investigation sensitivities across the clinical spectrum of sporadic Creutzfeldt-Jakob disease. Brain. (2006) 129:2278-87. doi: 10.1093/brain/awl159

8. Gao C, Shi Q, Tian C, Chen C, Han J, Zhou W, et al. The epidemiological, clinical, and laboratory features of sporadic Creutzfeldt-Jakob disease patients in China: surveillance data from 2006 to 2010. PLoS ONE. (2011) 6:e24231. doi: 10.1371/journal.pone.0024231

9. Shi Q, Gao C, Zhou W, Zhang B-Y, Chen J-M, Tian C, et al. Surveillance for Creutzfeldt-Jakob disease in China from 2006 to 2007. BMC Public Health. (2008) 8:360. doi: 10.1186/1471-2458-8-360

10. Lu C-J, Sun Y, Chen S-S. Incidence of Creutzfeldt-Jakob disease in Taiwan: a prospective 10-year surveillance. Eur J Epidemiol. (2010) 25:341-7. doi: 10.1007/s10654-010-9446-4

11. Vorberg IM, Chen C, Wang J-C, Shi Q, Zhou W, Zhang X-M, et al. Analyses of the survival time and the influencing factors of Chinese patients with prion diseases based on the surveillance data from 2008-2011. PLoS ONE. (2013) 8:e62553. doi: 10.1371/journal.pone.0062553

12. Yang J, Kuang H, Wang Q, Liu J, Chen X, Shang H. Analysis of Chinese patients with sporadic Creutzfeldt-Jakob disease. Prion. (2020) 14:137-42. doi: 10.1080/19336896.2020.1761515

13. Zhan W, Zhao W, Zhang J-T, Xing X-W, Huang D-H, Tian C$\mathrm{L}$, et al. Chinese specific characteristics of sporadic Creutzfeldt-Jakob disease: a retrospective analysis of 57 cases. PLoS ONE. (2013) 8:e58442. doi: 10.1371/journal.pone.0058442

14. Qi C, Zhang JT, Zhao W, Xing XW, Yu SY. Sporadic Creutzfeldt-Jakob disease: a retrospective analysis of 104 cases. Eur Neurol. (2020) 83:65-72. doi: 10.1159/000507189

15. Hermann P, Laux M, Glatzel M, Matschke J, Knipper T, Goebel $\mathrm{S}$, et al. Validation and utilization of amended diagnostic criteria in Creutzfeldt-Jakob disease surveillance. Neurology. (2018) 91:e331-e8. doi: 10.1212/WNL.0000000000005860

16. Nakatani E, Nishimura T, Zhou B, Kaneda H, Teramukai S, Nagai $\mathrm{Y}$, et al. Temporal and regional variations in sporadic CreutzfeldtJakob disease in Japan, 2001-2010. Epidemiol Infect. (2015) 143:1073-8. doi: 10.1017/S0950268814001605

17. Appleby BS, Rincon-Beardsley TD, Appleby KK, Wallin MT. Racial and ethnic differences in individuals with sporadic Creutzfeldt-jakob disease in the United States of America. PLoS ONE. (2012) 7:e38884. doi: 10.1371/journal.pone.0038884

18. Park SY, Wang MJ, Jang J-W, Park YH, Lim J-S, Youn YC, et al. The clinical stages of sporadic Creutzfeldt-Jakob disease with met/met genotype in korean patients. Eur Neurol. (2016) 75:213-22. doi: 10.1159/000445768

19. Sanjo N, Mizusawa H. Prion disease-the characteristics and diagnostic points in Japan. Clin Neurol. (2010) 50:287-300. doi: 10.5692/clinicalneurol.50.287

20. Kotkowski E, Cabot JH, Lacci JV, Payne DH, Cavazos JE, Romero RS, et al. Creutzfeldt-Jakob disease: in-hospital demographics report of national data in the United States from 2016 and review of a rapidly-progressive case. Clin Neurol Neurosurg. (2020) 197:106103. doi: 10.1016/j.clineuro.2020.106103

21. Llorens F, Rübsamen N, Hermann P, Schmitz M, Villar-Piqué A, Goebel S, et al. A prognostic model for overall survival in sporadic Creutzfeldt-Jakob disease. Alzheimers Dementia. (2020) 16:1438-47. doi: 10.1002/alz.12133

22. Pocchiari M. Predictors of survival in sporadic Creutzfeldt-Jakob disease and other human transmissible spongiform encephalopathies. Brain. (2004) 127:2348-59. doi: 10.1093/brain/awh249

23. Staffaroni A, Kramer A, Casey M, Kang H, Rojas J, Orrú C, et al. Association of blood and cerebrospinal fluid tau level and other biomarkers with survival time in sporadic Creutzfeldt-Jakob disease. JAMA Neurol. (2019) 76:969-77. doi: 10.1001/jamaneurol.2019.1071

24. Abu-Rumeileh S, Baiardi S, Ladogana A, Zenesini C, Bartoletti-Stella A, Poleggi A, et al. Comparison between plasma and cerebrospinal fluid biomarkers for the early diagnosis and association with survival in prion disease. J Neurol Neurosurg Psychiatry. (2020) 91:1181-8. doi: 10.1136/jnnp-2020-323826
25. Jeong B, Lee K, Kim N, Jin J, Kim J, Carp R, et al. Association of sporadic Creutzfeldt-Jakob disease with homozygous genotypes at PRNP codons 129 and 219 in the Korean population. Neurogenetics. (2005) 6:229-32. doi: 10.1007/s10048-005-0016-y

26. Nagoshi K, Sadakane A, Nakamura Y, Yamada M, Mizusawa H. Duration of prion disease is longer in japan than in other countries. J Epidemiol. (2011) 21:255-62. doi: 10.2188/jea.JE20100085

27. Chen S, He S, Xu Y, Teng H, Zhang J. Clinical presentation of sporadic Creutzfeldt-Jakob disease in Han-Chinese. Alzheimer Dis Assoc Disord. (2020) 34:188-90. doi: 10.1097/WAD.0000000000000350

28. Hamaguchi T, Sanjo N, Ae R, Nakamura Y, Sakai K, Takao M, et al. MM2-type sporadic Creutzfeldt-Jakob disease: new diagnostic criteria for MM2-cortical type. J Neurol Neurosurg Psychiatry. (2020) 91:1158-65. doi: 10.1136/jnnp-2020-323231

29. Krasnianski A, Meissner B, Schulz-Schaeffer W, Kallenberg K, Bartl M, Heinemann $U$, et al. Clinical features and diagnosis of the MM2 cortical subtype of sporadic Creutzfeldt-Jakob disease. Arch Neurol. (2006) 63:876-80. doi: 10.1001/archneur.63.6.876

30. Abu-Rumeileh S, Redaelli V, Baiardi S, Mackenzie G, Windl O, Ritchie D, et al. Sporadic fatal insomnia in Europe: phenotypic features and diagnostic challenges. Ann Neurol. (2018) 84:347-60. doi: 10.1002/ana.25300

31. Iwasaki Y, Akagi A, Mimuro M, Kitamoto T, Yoshida M. Factors influencing the survival period in Japanese patients with sporadic Creutzfeldt-Jakob disease. J Neurol Sci. (2015) 357:63-8. doi: 10.1016/j.jns.2015.06.065

32. Iwasaki Y, Mimuro M, Yoshida M, Kitamoto T, Hashizume Y. Survival to akinetic mutism state in Japanese cases of MM1-type sporadic CreutzfeldtJakob disease is similar to Caucasians. Eur J Neurol. (2011) 18:999-1002. doi: 10.1111/j.1468-1331.2010.03185.x

33. Krasnianski A, Kaune J, Jung K, Kretzschmar H, Zerr I. First symptom and initial diagnosis in sporadic CJD patients in Germany. J Neurol. (2014) 261:1811-7. doi: 10.1007/s00415-014-7410-Z

34. Rabinovici GD, Wang PN, Levin J, Cook L, Pravdin M, Davis J, et al. First symptom in sporadic Creutzfeldt-Jakob disease. Neurology. (2006) 66:286-7. doi: 10.1212/01.wnl.0000196440.00297.67

35. Steinhoff B, Zerr I, Glatting M, Schulz-Schaeffer W, Poser S, Kretzschmar H. Diagnostic value of periodic complexes in Creutzfeldt-Jakob disease. Ann Neurol. (2004) 56:702-8. doi: 10.1002/ana.20261

36. Bortone E, Bettoni L, Giorgi C, Terzano MG, Trabattoni GR, Mancia D. Reliability of EEG in the diagnosis of Creutzfeldt-Jakob disease. Electroencephalogr Clin Neurophysiol. (1994) 90:323-30. doi: 10.1016/0013-4694(94)90048-5

37. Chiofalo N, Fuentes A, Gálvez S. Serial EEG findings in 27 cases of Creutzfeldt-Jakob disease. Arch Neurol. (1980) 37:143-5. doi: 10.1001/archneur.1980.00500520041005

38. Primavera A, Tabaton M, Leonardi A. Periodic lateralized discharges in Creutzfeldt-Jakob disease: serial electroencephalographic studies. Revue d'electroencephalographie et de neurophysiologie clinique. (1984) 13:379-82. doi: 10.1016/S0370-4475(84)80045-3

39. Burger LJ, Rowan AJ, Goldensohn ES. Creutzfeldt-Jakob disease. An electroencephalographic study. Arch Neurol. (1972) 26:428-33. doi: 10.1001/archneur.1972.00490110062006

40. Steinhoff B, Räcker S, Herrendorf G, Poser S, Grosche S, Zerr I, et al. Accuracy and reliability of periodic sharp wave complexes in Creutzfeldt-Jakob disease. Arch Neurol. (1996) 53:162-6. doi: 10.1001/archneur.1996.005500200 74017

41. Tschampa H, Neumann M, Zerr I, Henkel K, Schröter A, Schulz-Schaeffer W, et al. Patients with Alzheimer's disease and dementia with Lewy bodies mistaken for Creutzfeldt-Jakob disease. J Neurol Neurosurg Psychiatry. (2001) 71:33-9. doi: 10.1136/jnnp.71.1.33

42. Muayqil T, Gronseth G, Camicioli R. Evidence-based guideline: diagnostic accuracy of CSF 14-3-3 protein in sporadic CreutzfeldtJakob disease: report of the guideline development subcommittee of the American Academy of Neurology. Neurology. (2012) 79:1499-506. doi: 10.1212/WNL.0b013e31826d5fc3

43. Shiga Y, Miyazawa K, Sato S, Fukushima R, Shibuya S, Sato Y, et al. Diffusion-weighted MRI abnormalities as an early diagnostic marker for Creutzfeldt-Jakob disease. Neurology. (2004) 63:443-9. doi: 10.1212/01.WNL.0000134555.59460.5D 
44. Young GS, Geschwind MD, Fischbein NJ, Martindale JL, Henry RG, Liu S, et al. Diffusion-weighted and fluid-attenuated inversion recovery imaging in Creutzfeldt-Jakob disease: high sensitivity and specificity for diagnosis. Am J Neuroradiol. (2005) 26:1551-62.

45. Vitali P, Maccagnano E, Caverzasi E, Henry R, Haman A, Torres-Chae C, et al. Diffusion-weighted MRI hyperintensity patterns differentiate CJD from other rapid dementias. Neurology. (2011) 76:1711-9. doi: 10.1212/WNL.0b013e31821a4439

46. Yi S, Park K, Yoon S, Kim E, Shin W. Relationship between clinical course and diffusion-weighted MRI findings in sporadic Creutzfeldt-Jakob Disease. Neurol Sci. (2008) 29:251-5. doi: 10.1007/s10072-008-0976-4

47. Gao T, Lyu J, Zhang J, Lou X, Zhao W, Xing X, et al. Diffusion-weighted MRI findings and clinical correlations in sporadic Creutzfeldt-Jakob disease. $J$ Neurol. (2015) 262:1440-6. doi: 10.1007/s00415-015-7723-6

48. Hamlin C, Puoti G, Berri S, Sting E, Harris C, Cohen M, et al. A comparison of tau and 14-3-3 protein in the diagnosis of Creutzfeldt-Jakob disease. Neurology. (2012) 79:547-52. doi: 10.1212/WNL.0b013e318263565f

49. Lattanzio F, Abu-Rumeileh S, Franceschini A, Kai H, Amore G, Poggiolini I, et al. Prion-specific and surrogate CSF biomarkers in Creutzfeldt-Jakob disease: diagnostic accuracy in relation to molecular subtypes and analysis of neuropathological correlates of p-tau and A $\beta 42$ levels. Acta Neuropathol. (2017) 133:559-78. doi: 10.1007/s00401-017-1683-0

50. Abu-Rumeileh S, Baiardi S, Polischi B, Mammana A, Franceschini A, Green A, et al. Diagnostic value of surrogate CSF biomarkers for CreutzfeldtJakob disease in the era of RT-QuIC. J Neurol. (2019) 266:3136-43. doi: 10.1007/s00415-019-09537-0

51. Skillbäck T, Rosén C, Asztely F, Mattsson N, Blennow K, Zetterberg H. Diagnostic performance of cerebrospinal fluid total tau and phosphorylated tau in Creutzfeldt-Jakob disease: results from the Swedish Mortality Registry. JAMA Neurol. (2014) 71:476-83. doi: 10.1001/jamaneurol.2013.6455

52. Riemenschneider M, Wagenpfeil S, Vanderstichele H, Otto M, Wiltfang J, Kretzschmar H, et al. Phospho-tau/total tau ratio in cerebrospinal fluid discriminates Creutzfeldt-Jakob disease from other dementias. Mol Psychiatry. (2003) 8:343-7. doi: 10.1038/sj.mp.4001220

53. Foutz A, Appleby B, Hamlin C, Liu X, Yang S, Cohen Y, et al. Diagnostic and prognostic value of human prion detection in cerebrospinal fluid. Ann Neurol. (2017) 81:79-92. doi: 10.1002/ana.24833

54. McGuire L, Peden A, Orrú C, Wilham J, Appleford N, Mallinson G, et al. Real time quaking-induced conversion analysis of cerebrospinal fluid in sporadic Creutzfeldt-Jakob disease. Ann Neurol. (2012) 72:278-85. doi: 10.1002/ana.23589
55. Green AJE. RT-QuIC: a new test for sporadic CJD. Pract Neurol. (2019) 19:49-55. doi: 10.1136/practneurol-2018-001935

56. Rhoads D, Wrona A, Foutz A, Blevins J, Glisic K, Person M, et al. Diagnosis of prion diseases by RT-QuIC results in improved surveillance. Neurology. (2020) 95:e1017-e26. doi: 10.1212/WNL.0000000000010086

57. Gelpi E, Heinzl H, Hoftberger R, Unterberger U, Strobel T, Voigtlander $\mathrm{T}$, et al. Creutzfeldt-Jakob disease in Austria: an autopsy-controlled study. Neuroepidemiology. (2008) 30:215-21. doi: 10.1159/0001 26915

58. Sánchez-González L, Maddox RA, Lewis LC, Blevins JE, Harker EJ, Appleby BS, et al. Human prion disease surveillance in Washington State, 2006-2017. JAMA Network Open. (2020) 3:e2020690. doi: 10.1001/jamanetworkopen.2020.20690

59. Heinemann U, Krasnianski A, Meissner B, Varges D, Kallenberg K, SchulzSchaeffer WJ, et al. Creutzfeldt-Jakob disease in Germany: a prospective 12-year surveillance. Brain. (2007) 130:1350-9. doi: 10.1093/brain/awm063

60. Stehmann C, Senesi M, Lewis V, Ummi M, Simpson M, Klug G, et al. Creutzfeldt-Jakob disease surveillance in Australia: update to 31 December 2018. Commun Dis Intellig. (2019) 43:1-18. doi: 10.33321/cdi.2019.43.35

61. Litzroth A, Cras P, De Vil B, Quoilin S. Overview and evaluation of 15 years of Creutzfeldt-Jakob disease surveillance in Belgium, 1998-2012. BMC Neurol. (2015) 15:250. doi: 10.1186/s12883-015-0507-x

Conflict of Interest: The authors declare that the research was conducted in the absence of any commercial or financial relationships that could be construed as a potential conflict of interest.

Publisher's Note: All claims expressed in this article are solely those of the authors and do not necessarily represent those of their affiliated organizations, or those of the publisher, the editors and the reviewers. Any product that may be evaluated in this article, or claim that may be made by its manufacturer, is not guaranteed or endorsed by the publisher.

Copyright () 2021 Feng, Zhao, Zhou, Ye, Yu, Jiang, Deng, Zhou, Ma, Shan and Zhou. This is an open-access article distributed under the terms of the Creative Commons Attribution License (CC BY). The use, distribution or reproduction in other forums is permitted, provided the original author(s) and the copyright owner(s) are credited and that the original publication in this journal is cited, in accordance with accepted academic practice. No use, distribution or reproduction is permitted which does not comply with these terms. 